\title{
Spatio-Temporal Clustering of Traffic Data with Deep Embedded Clustering
}

\author{
Reza Asadi \\ rasadi@uci.edu \\ PhD Candidate, Department of Computer Science \\ University of California Irvine \\ Irvine, California
}

\begin{abstract}
Traffic data is a challenging spatio-temporal data, and a multivariate time series data with spatial similarities. Clustering of traffic data is a fundamental tool for various machine learning tasks including anomaly detection, missing data imputation and short term forecasting problems. In this paper, first, we formulate a spatiotemporal clustering problem and define temporal and spatial clusters. Then, we propose an approach for finding temporal and spatial clusters with a deep embedded clustering model. The proposed approach is examined on traffic flow data. In the analysis, we present the properties of clusters and patterns in the dataset. The analysis shows that the temporal and spatial clusters have meaningful relationships with temporal and spatial patterns in traffic data, and the clustering method effectively finds similarities in traffic data.
\end{abstract}

\section{CCS CONCEPTS}

- Information systems $\rightarrow$ Spatial-temporal systems; • Computing methodologies $\rightarrow$ Machine learning; Neural networks.

\section{KEYWORDS}

Neural Networks, Time Series Clustering,

Spatio-temporal data, Traffic Flow Data

\section{ACM Reference Format:}

Reza Asadi and Amelia Regan. 2019. Spatio-Temporal Clustering of Traffic Data with Deep Embedded Clustering. In 3rd ACM SIGSPATIAL International Workshop on Prediction of Human Mobility (PredictGIS'19), November 5, 2019, Chicago, IL, USA. ACM, New York, NY, USA, 8 pages. https://doi.org/10. $1145 / 3356995.3364537$

\section{INTRODUCTION}

The volume of traffic flow data has increased with the advent of new sensing technologies. In most transportation systems, a large number of sensors gather traffic data in a geographical area. The

Permission to make digital or hard copies of all or part of this work for personal or classroom use is granted without fee provided that copies are not made or distributed for profit or commercial advantage and that copies bear this notice and the full citation on the first page. Copyrights for components of this work owned by others than the author(s) must be honored. Abstracting with credit is permitted. To copy otherwise, or republish, to post on servers or to redistribute to lists, requires prior specific permission and/or a fee. Request permissions from permissions@acm.org.

PredictGIS'19, November 5, 2019, Chicago, IL, USA

(C) 2019 Copyright held by the owner/author(s). Publication rights licensed to ACM. ACM ISBN 978-1-4503-6964-0/19/11 ..\$15.00

https://doi.org/10.1145/3356995.3364537

\author{
Amelia Regan \\ aregan@uci.edu \\ Professor, Department of Computer Science \\ University of California Irvine \\ Irvine, California
}

data are gathered over specific time periods, and it has both spatial and temporal patterns. Traffic data is considered a high-dimensional time series data, which include the flow, speed and occupancy of a large number of sensors, and in which there is a spatial correlation among neighboring time series. The problem is challenging because of both temporal and spatial patterns in data. Spatio-temporal data arise in broad areas of engineering and environmental sciences. In [1], they discuss various types of spatio-temporal data and their corresponding data mining approaches. In transportation systems, traffic flow data is a challenging spatio-temporal data. Analyzing such data can improve the performance of intelligent transportation systems, reduce traffic congestion, and air pollution [2]. Also, spatio-temporal congestion patterns is studied for traffic flow data [3]. However, the increasing volume of traffic data requires the development of large-scale machine learning algorithms and big data analytics [4]. Various studies examine data-driven approaches and large-scale machine learning techniques in transportation systems [5].

Exploring similarities in spatio-temporal patterns results in finding interesting patterns in data. Clustering algorithms not only find similarities among data points, but also explore deep insights in temporal and spatial patterns. Clustering algorithms have been used in a broad range of machine learning tasks. In this paper, we focus on developing a clustering method for spatio-temporal data. Here, we describe some of the important applications of using clustering method to solve machine learning problems. In [6], a clustering method is used for detecting anomalies. Data points which are far from center of clusters or are in separate clusters can be considered as anomalies. In [7], they use k-means clustering for finding similar spatial data. Then an autoencoder imputes missing data for each cluster. In [8], the spatial clusters are used to improve the performance of a forecasting problem. These works illustrate that the clustering of spatio-temporal data is a fundamental tool for various machine learning tasks.

Moreover, clustering algorithms have been used on various types of traffic flow data. In [9], they cluster vehicle trajectory data to model traffic flow. The data is obtained from traffic surveillance of intersections. In [10], they cluster traffic flow patterns based on dynamical traffic flow models. A macroscopic flow model is used with a multivariate clustering approach. However, most of traffic flow data are obtained by loop detectors or sampling from GPS data on road networks. Each road segment includes traffic data for a time stamp. Here, we focus on traffic data obtained by loop detectors. Clustering of traffic data is a common data mining analysis [11]. In [12], they propose a fuzzy clustering technique for traffic flow data. 
A Dynamic Time Warping (DTW) distance among traffic flow data is used as a similarity measure. Also, clustering of traffic data can find interesting insights in physical properties of traffic flow data. In [13], they apply fuzzy k-means clustering to analyze variations in traffic flow states. While clustering of traffic data is an important problem, it is a challenging one. Traffic data is spatio-temporal data in which there is a non-linear relation among spatial and temporal neighborhoods. Clustering of time series data requires a distance metric, such as DTW, which extracts a non-linear relation among time series data. Moreover, traffic data is a multi-variate time series with spatial correlation. Applying traditional clustering methods, such as k-means, on traffic data is computationally expensive and can have low performance [14], though more efficient heuristic methods for k-means clustering of traffic flow data have been studied [15].

Since traffic flow data is a multi-variate time series data, here we review some of the recent works for time series clustering. In [16], they describe a broad range of time series clustering applications. Also, the main components of time series clustering have been studied, including time series representations, similarity and distance measures, clustering prototypes and time series clustering. In [17], they describe the challenges of k-means clustering with time warp measure. They propose a weighted and kernel time warp measures for k-means clustering. Their method has a faster estimation of clusters. Further investigation in time series clustering is studied in [18].

Deep learning models significantly improve performance of various machine learning problems, such as computer vision and natural language processing. Such models have been broadly used for various large-scale spatio-temporal problems [19], [20]. Moreover, deep learning models for clustering tasks are broadly studied in [21] Deep embedded clustering is primarily introduced in [22]. Variations of the model have been studied in a broad domains. A joint training of the model to preserve the latent feature space structure is proposed [23]. In [24], they analyze a clustering-friendly latent representations, in which jointly optimize dimension reduction by a neural network and a k-means clustering. While most of the works apply deep embedded clustering on images, there are few studies to show their performance on time series data. In [25], they jointly cluster and train the model. They also segment time series data with agglomerative clustering.

The aforementioned works show the importance of developing a deep learning model for the spatio-temporal clustering problem. In addition, they show the recent advances in deep learning models for clustering tasks. However, there is lack of study to investigate the performance of deep learning models for spatio-temporal clustering. Here, we formulate and propose a procedure for spatio-temporal clustering with deep learning models. We define temporal and spatial clusters and analyze spatial and temporal clusters obtained by deep learning models in details. Such an analysis shows the existence of temporal and spatial patterns in the clusters. Also, we illustrate that the proposed approach can better finds temporal clusters, as it captures the non-linear relation among temporal data. Our focus in this study is on traffic flow data. Clustering of such data not only improves the performance of clustering based machine learning models, such as anomaly detection with a clustering method, but also provides opportunities to further investigate transportation systems using temporal and spatial clusters.

In section 2, we describe the technical background for the proposed approach. In section 3, we define spatial and temporal clusters, and describe the proposed approach for finding spatial clusters with temporal clusters. In section 4, a deep learning investigation of clustering on traffic flow data is illustrated. In Section 5 our conclusion and future work are presented.

\section{TECHNICAL BACKGROUND}

\subsection{Problem Definition}

Spatio-temporal data is represented with a matrix $\mathbf{X} \in \mathbb{R}^{\mathrm{s} \times \overline{\mathrm{t}} \times \mathrm{f}}$, where $s$ is the number of sensors, $\bar{t}$ is the number of time stamps and $f$ is the number of traffic features, including flow, speed and occupancy. Each sensor has its own time series data $\mathbf{x}_{i} \in \mathbb{R}^{\overline{\mathrm{t}} \times f}$. Clustering of whole time series is computationally expensive and finding temporal patterns in such clusters is not possible. Hence, we generate sub-sequences of time series data and apply clustering methods on them. A sliding window approach with time window $w$ generates a sequence of traffic data. A traffic state at time stamp $t$ and location $i$ is represented with $\mathbf{X}_{i}^{t} \in \mathbb{R}^{w \times f}$. In other words, the data points are represented with traffic states.

A clustering method assigns each traffic state $\mathbf{x}_{\mathrm{i}}^{\mathrm{t}}$ into a cluster $\mathbf{c}_{\mathbf{j}}$, where $\mathrm{j} \in\{1, \ldots,|\mathrm{C}|\}$, and $|\mathrm{C}|$ is the given number of clusters. For a latent representation of $\mathbf{x}_{\mathrm{i}}^{\mathrm{t}}$, represented with $\mathbf{z}_{\mathrm{i}}^{\mathrm{t}}$, the clustering method assigns $\mathbf{z}_{i}^{t}$ to the clusters. A temporal cluster consists of traffic states from all sensors. In other words, any traffic state can be a member of a temporal cluster. In algorithm 1, we find spatial clusters from temporal clusters. A spatial cluster includes some of the sensors. A spatial cluster is obtained based on similarity of temporal clusters. The similarity of two sensors is obtained based on the similarity of their temporal clusters. In this way, instead of comparing two sensors' data for a long time period, e.g. six of months of training data, we have temporal similarity for a short time windows with size w.

Similarity of time series cannot be obtained from the euclidean distance of their vectors. A non-linear distance function, e.g. DTW, can provide a similarity (distance) measure for two time series. Another approach is to find latent feature representations and use a euclidean distance measure on those representations, as the temporal relation does not necessarily hold in lower dimension space. We consider both approaches in this paper. We also examine this property of latent features in experimental results by finding the correlation between DTW distance of traffic states and euclidean distance of latent features. In such a way, the clustering is more efficient, as the distance function is applied on lower dimension space.

\subsection{Autoencoders}

An autoencoder is primarily proposed in [26]. It reconstructs the input data with $\overline{\mathbf{x}}=\mathrm{f}(\mathbf{x}, \boldsymbol{\theta})$, given model parameters $\boldsymbol{\theta}$, an input data $\mathbf{x}$ and reconstructed input $\overline{\mathbf{x}}$. An encoder is the first neural network component, which reduces the dimension of input data to 
a latent feature space $\mathbf{h} \in \mathbb{R}^{\mathrm{d}}$, where $\mathrm{d}<\mathrm{n}$. Latent features consists of the most important patterns of input data. The second neural network component is a decoder, which reconstructs the input data from its latent representation.

$$
\begin{aligned}
& \mathbf{h}=\sigma\left(\operatorname{drop}(\mathbf{x}) \mathbf{w}^{1}+\mathbf{b}^{1}\right) \\
& \overline{\mathbf{x}}=\sigma\left(\operatorname{drop}(\mathbf{h}) \mathbf{w}^{2}+\mathbf{b}^{2}\right)
\end{aligned}
$$

where the activation function and the dropout function are represented with $\sigma($.$) and drop(.), respectively. In a deep autoencdoer,$ the encoder considers several layers and reduces the input dimension into latent feature space $\mathbf{h}$. Then the decoder as a multi layer neural network, reconstructs the input. The loss function $L(\mathbf{x}, \overline{\mathbf{x}})$, e.g. mean square error, reduces the difference of input data $\mathbf{x}$ and its reconstruction $\overline{\mathbf{x}}$.

\subsection{Deep Embedded Clustering}

A Deep embedded clustering neural network is introduced in [22]. The encoder transforms $\mathbf{x}$ into latent feature space $\mathbf{z}$. The clustering layer is connected to latent feature layer. The weights of clustering layer is initialized with cluster centers obtained by k-means clustering. Cluster center $i$ is represented with $\mu_{i} \in \mathbb{R}^{d}$. Given $k$ as the number of clusters, and $d$ as latent feature size, the clustering layer is represented with a dense layer $\mathbb{R}^{\mathrm{d}} \rightarrow \mathbb{R}^{\mathrm{k}}$. In other words, it converts latent features into a vector of size $k$, which k-th element represents the probability that the data point is assigned to the cluster $\mathrm{k}$.

Given initial cluster centers $\left\{\mu_{1}, \ldots, \mu_{k}\right\}$ and latent features $\mathbf{z}$, a student's t-distribution measures the similarity between cluster centers $\mu_{\mathrm{i}}$ and data points $\mathbf{x}_{\mathbf{i}}$ as follows,

$$
q_{i j}=\frac{\left(1+\left\|z_{i}-\mu_{j}\right\|^{2}\right)^{-1}}{\sum_{k}\left(1+\left\|z_{i}-\mu_{k}\right\|^{2}\right)^{-1}}
$$

where the degree of freedom of the student's t-distribution is one. The probability of assigning data point $\mathbf{x}_{\mathrm{i}}$ to cluster $\mu_{\mathrm{j}}$ is represented with $\mathrm{q}_{\mathrm{ij}}$. The assigned cluster is $\operatorname{argmax}_{\mathrm{j}} \mathbf{q}_{\mathrm{ij}}$. The clustering algorithm iteratively adjusts clusters by learning from high confidence assignments. To learn from high confidence assignments, an auxiliary target distribution $\mathrm{p}_{\mathrm{ij}}$ is as follows,

$$
p_{i j}=\frac{q_{i j}^{2} / f_{j}}{\sum_{k} q_{i j}^{2} / f_{k}},
$$

KL-divergence loss between $\mathrm{q}_{\mathrm{ij}}$ and $\mathrm{p}_{\mathrm{ij}}$ learns the high confidence soft cluster assignment,

$$
K L(P \| Q)=\sum_{i} \sum_{j} p_{i j} \log \frac{p_{i j}}{q_{i j}}
$$

A joint training of autoencoders with reconstruction loss and clustering loss is proposed in [23], in which an autoencoder reconstructs input and cluster data points simultaneously. A joint training preserves the structure of latent feature representation of data points. In this paper, we pretrain an autoencoder with traffic states and using a joint training method we obtain the clusters of temporal data.

\section{SPATIO-TEMPORAL CLUSTERING METHOD}

Here, we describe the proposed method for spatio-temporal clustering of traffic data.

As represented in Section 2.1, we consider the clustering of traffic states $\mathbf{x}_{i}^{t} \in \mathbb{R}^{\mathrm{w} \times \mathrm{f}}$ for a given time window $w$ and features $f$. While in alternative approaches, one can consider the problem of clustering a whole time series $\mathbf{x}_{\mathbf{i}} \in \mathbb{R}^{\overline{\mathrm{t}} \times \mathrm{f}}$, or sub sequences of spatio-temporal data $\mathbf{x}^{\mathrm{t}} \in \mathbb{R}^{\mathrm{s} \times \mathrm{w} \times \mathrm{f}}$. Clustering of traffic states provides more flexibility and efficiency. First, we can have multiple clusters for one sensor in different time stamps, unlike clustering of whole time series. Second, we can cluster sensors independent of one other, unlike clustering of sub sequences of spatio-temporal data. However, in this approach, we need a method to obtain temporal and spatial clusters. In this section, we describe our proposed method.

The input of autoencoder consists of all traffic states $\mathbf{x}_{i}^{t}$ for all sensors $i$ and time stamps $t$. The autoencoder reconstructs traffic states. Also, the encoder outputs their latent feature representations. The deep embedded clustering assigns the latent feature of each traffic state to the clusters. The probability that each traffic state would be in cluster $k$ is represented with $c_{i k}^{t}$. The assigned cluster would be obtained by $c_{i}^{t}=\operatorname{argmax}_{k} c_{i k}^{t}$. We represent the temporal cluster $c_{i}^{t}$ for sensor $i$ and time stamp t. Although temporal clusters can find meaningful patterns in data, spatial clustering is useful to find the relation of sensors in a geographical area. Spatial clustering needs aggregation of temporal clusters. First, we define spatial similarity as the similarity of temporal clusters for a time interval. In other words, spatial similarity of two sensors $i$ and $j$ for a time time interval $\mathbf{T}$ is the number of times their temporal clusters are equal for $t \in \mathbf{T}$. For example, if the objective is to find spatial similarity of sensors between $3 \mathrm{pm}$ to $5 \mathrm{pm}$ on one day, then it can be obtained by comparing $c_{i}^{t}$ and $c_{j}^{t}$ for all $t$ in range of $3 p m$ to $5 \mathrm{pm}$. In this approach, the spatial clustering is dynamic over various time intervals. Spatial clustering can be obtained based on these temporal clusters. In the rest of this section we describe the proposed procedure.

The proposed procedure is in Algorithm 1. The input of spatiotemporal data is represented with $\mathbf{x} \in \mathbb{R}^{s \times \overline{\mathrm{t}} \times \mathrm{k}}$. First we normalize data with Normalize(.). All of the features are normalized in the range of zero to one over time stamps to have a same maximum and minimum value. A sliding window method SlidingWindow(., .) receives input data and window size of $w$. A sequence of $\mathbf{x}^{\mathrm{t}} \in \mathbb{R}^{s \times w \times k}$ is generated for $\{0, \ldots, \overline{\mathrm{t}}\}$. An autoencoder trainAutoencoder(.) receives an input traffic states $\mathbf{x}_{i}^{t}$ for all sensors $i$ and time stamp $t$. It reconstructs each input data and train model parameters. The model parameters are stored in modelPre. Since the autoencoder has encoder-decoder architecture, it can output the latent feature representation of input data with predictLatent(.), as the output of layer $\mathbf{z}$ with lowest dimension layer.

The first step of applying deep embedded clustering is to find initial clusters as introduced in [22]. A k-means clustering initialKmeans(.) is applied on latent feature representations for few iterations. The 


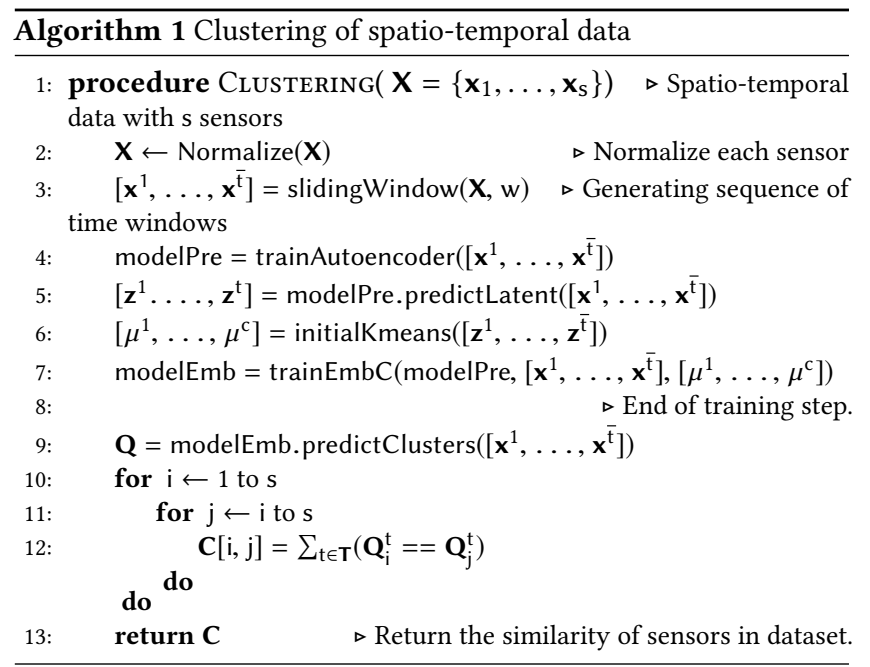

output is the initial cluster means. For a given number of clusters c, the mean of clusters is found, represented with $\boldsymbol{\mu}_{\mathrm{k}}$ for cluster $\mathrm{k}$. The deep embedded clustering trainEmbeddedC(.,.,.) receives the pre-trained autoencoder, input data and mean of clusters. The model has one clustering layer connected to latent feature layer, further details were provided in Section 2.3 of this paper. The output of deep embedded clustering is $\mathbf{Q} \in \mathbb{R}^{\bar{t} \times s, c}$, which includes the probability of membership of each traffic state $\mathbf{x}_{\mathrm{i}}^{\mathrm{t}}$ to the clusters c clusters. For all sensors in dataset, the number of times two sensors are in same clusters is considered as their spatial similarity, which is obtained in lines 9-13 of Algorithm 1. This comparison is for all $t$ in time interval T. In other words, for each time interval, we can obtain spatial similarity among sensors. Such a value is stored in $\mathbf{C}$. Since the value of similarity matrix $\mathbf{C}^{\mathbf{T}}$ depends on time interval $\mathbf{T}$, we can obtain different spatial similarities over various times of day. To find spatial clusters from $\mathbf{C}^{\mathbf{T}}$, a threshold value $\epsilon$ on each element $\mathbf{C}_{\mathrm{ij}}^{\mathbf{T}}$ can finds the clusters. In other words, if we have $\mathbf{C}_{\mathrm{ij}}^{\mathbf{T}}>\epsilon$, then two sensors $i$ and $j$ are in a same cluster. In the experimental results, we illustrate the output similarity matrix $\mathbf{C}$ using heatmaps and spatial clusters obtained by Algorithm 1. This approach for finding spatial clusters has advantages over applying a clustering method on spatio-temporal data directly. Our objective is to find a dynamic spatial cluster set. In such a way, we can obtain various spatial clustering in different time intervals.

\section{EXPERIMENTAL RESULTS}

Here we illustrate the results for clustering of traffic flow data. The objective is to show the existence of spatial and temporal patterns in the found clusters.

The deep learning model is implemented with Keras. We use a fullyconnected autoneocder with 7 layers. All of the layers have Relu activation function and dropout rate of 0.2 . The number of hidden units are $(32,32,128,4,128,32,32)$ in seven fully-connected layers. The batch size of 288, one day with 5-min time stamp, and Adam optimizer are selected. The deep embedded clustering layer has a $I_{2}$ regularization of 0.1 . The loss function in joint training of the model is 0.05 for clustering loss term, the KL divergence of clustering layer's output and auxiliary target, and 1.0 for reconstruction loss term. The higher value of reconstruction loss keeps the structure of latent feature space. The joint training of clustering layer with reconstruction loss has 5000 epochs, and every 50 epochs we update the auxiliary target distribution $\mathbf{p}$ with high confidence soft cluster assignments.

\subsection{Data}

Traffic data are selected from the CalTrans PeMS data[27], which have widely used by researchers examining large-scale traffic flow models. Traffic data is gathered from main-line loop detector sensors every 30 seconds and aggregated to every 5 minutes. The data is for US-101 South highway, in the Bay Area of California, which includes 26 mainline sensors, illustrated in Fig. 1. We select the data for the first two months of 2016. The data includes flow, speed and occupancy. In a preprocessing step, we re-scale data into the range of $[0,1]$. Since the data is not stationary, the output of clustering can be meaningless and dependent on the hours of a day. We subtract each time window of size $w$ from its first element. After hyper parameter tuning, a time window of size 12, one hour, is selected. In such a way, we expect the model to cluster time series data based on the slope, periodic patterns and non-linear temporal similarities.

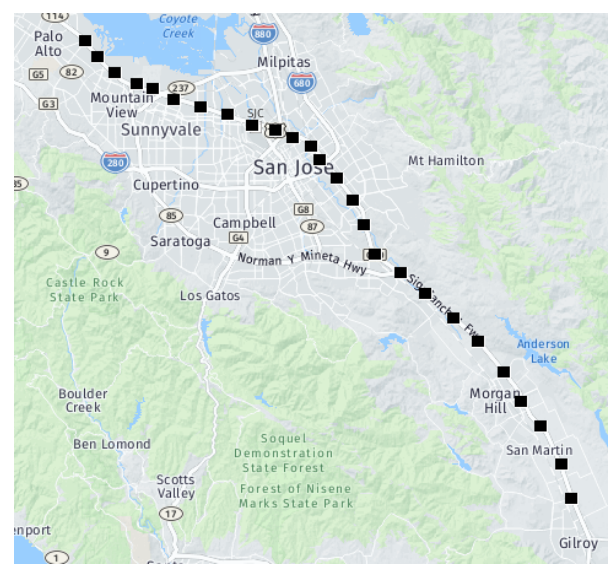

Figure 1: 26 sensors on one highway in Bay Area, California are selected. The black boxes are the main-line loop detector sensors.

\subsection{Temporal Clusters}

The latent feature representation of one sensor's traffic states are represented in Fig 2. A t-distributed stochastic neighbor embedding (TSNE) introduced in [28], method is used for showing latent features in two dimension space. The color of each traffic state represents the hours of a day. One day is grouped into 10 colors, every 2.4 hours. The plot is for five weekdays. The results show that different time stamps are distinguishable from each other, e.g., all of the data points around $3 \mathrm{pm}$ are in same area. While the latent feature representation shows that there is a temporal pattern in data, we need to examine the similarity of time series based on latent feature representation. In Fig 3a, we select three areas and two data points from each area. The corresponding traffic flow is 
represented in Fig. 3b, 3c and 3d. To simplify the figures, we only present traffic flow, and ignore traffic occupancy and speed. The similarity between green and red lines represents the non-linear similarity between two closest data points. The figures clearly illustrate the meaningful relation among time series data and their corresponding latent features. In other words, the slope and nonlinear temporal relation exists in the samples. Also, we note that as it is illustrated in Section 4.1 (Data), all of the data points are subtracted from their first element to make them stationary, hence the first element is zero in all of the plots.

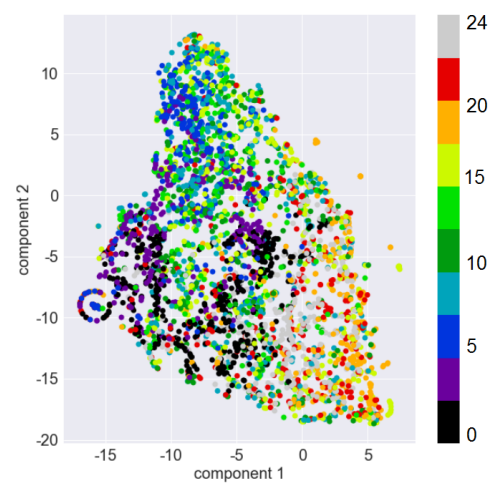

Figure 2: TSNE representation of autoencoder's latent features.

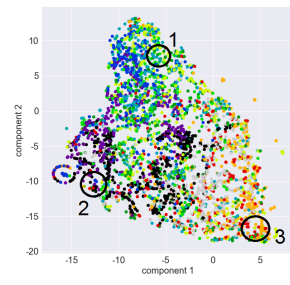

(a)

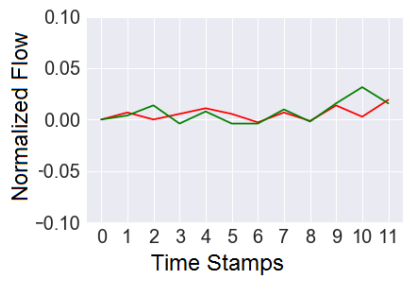

(c)

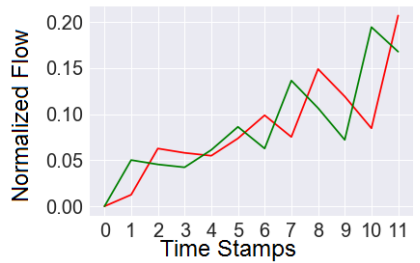

(b)

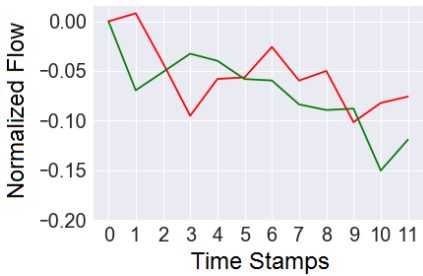

(d)
Figure 3: To represent the relation between latent features and time series data points, two data points are selected from three regions 1, 2 and 3 in Fig.a and represented in Fig.b, Fig.c and Fig.d.

Another interesting insight in temporal clusters is to find the relation among the clusters, and their DTW distance. In Fig 4, the relation between DTW and latent features is represented. For any

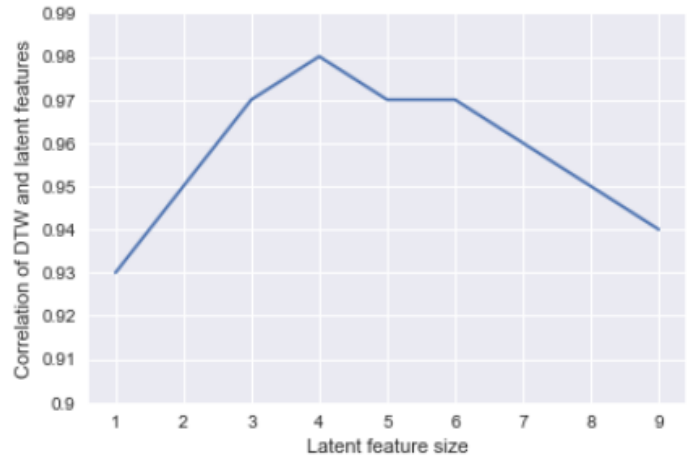

Figure 4: The plot for the relation of DTW distance and latent feature space.

given data points, the euclidean distance of latent features is calculated. Also the DTW among their time series is calculated. The correlation between euclidean distance of latent features and DTW of time series is presented in the Figure 4 . Such an analysis illustrates that latent feature representation has a direct relation with DTW. The correlation between latent features and dynamic time warping is obtained and illustrated in Fig 4. The figure shows the changes of DTW for various sizes of latent features. Such an analysis shows that with a latent feature size of 4 , the correlation between dynamic time warping and euclidean distance of latent features is 0.98 . Such a high value of correlation shows the direct relation between them. A K-means clustering algorithm has a $\mathrm{O}\left(\mathrm{n}^{2}\right)$ computational time. Using a DTW method on time windows of size $\mathrm{w}$, the total computation would be $\mathrm{O}\left(\mathrm{n}^{2} \mathrm{w}^{2}\right)$. On the other hand, euclidean distance of two vectors with size of I has I comparisons. Then, computational time of K-means on latent feature space with euclidean distance would be $\mathrm{O}\left(\mathrm{n}^{2} \mathrm{I}\right)$, where latent feature size $\mathrm{I}$ is very smaller than original size of time series $w$. This reduction in computational time of $\mathrm{k}$-means clustering and high correlation between euclidean distance of latent feature representations and dynamic time warping is our first conclusion on the dataset.

The analysis in this section illustrates that latent features of traffic states keeps the non-linear relation among temporal data. A deep embedded clustering model uses the clustering of latent features. In next, we illustrate the patterns in output of deep embedded clustering model.

Deep embedded clustering uses latent feature representation of time series to find their clusters. The output of clustering layer is a vector $\mathbf{c} \in \mathbb{R}^{\mathrm{K}}$, where $\mathbf{c}_{\mathrm{k}}$ is the probability that a data point assigned to the cluster $\mathrm{k}$. To analyze the patterns, we use tsne for representing the vector $\mathbf{c}$ over different hours in Fig 5. The results show that different hours have their own clustering patterns and the cluster assignment are separable based on time stamp. This figure shows that not only latent features have distinguishable temporal patterns, but also cluster probabilities are distinguishable over different time stamps.

In clustering algorithms, finding an appropriate number of clusters is a challenging task. In spatio-temporal data, data have both context of time and space. We cannot easily estimate the number of clusters 


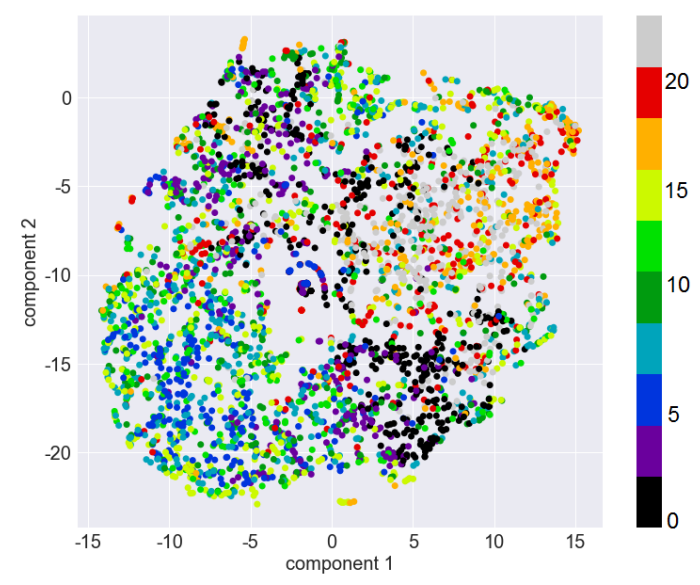

Figure 5: TSNE of cluster probabilities as the output of deep embedded clustering.

for temporal data. In deep embedded clustering, the number of initial clusters should be given. To obtain an appropriate number of clusters in k-means clustering, a general approach is to use inertia, as the sum of squares of data points to their cluster centers. In Fig 6, the plot shows the value of inertia for the given number of clusters. The results show that the optimum number of clusters is around 70 . The optimum value can be obtained, when the reduction in inertia becomes linear.

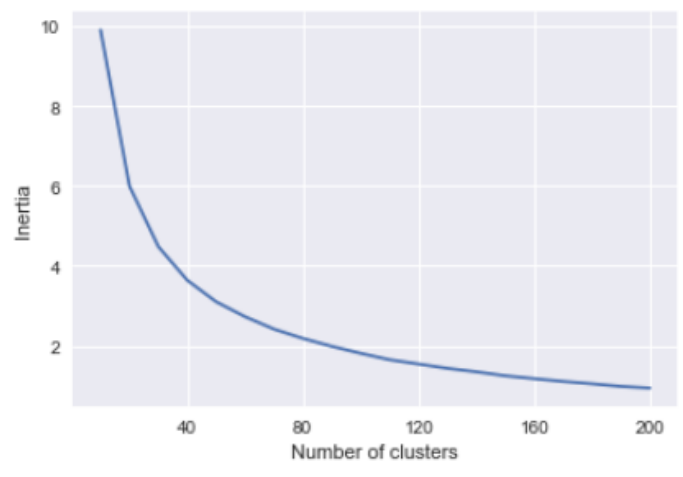

Figure 6: The plot of sum of squares of data points to cluster centers in terms of the number of clusters.

The histogram of the size of clusters is represented in Fig 7. The histogram shows the size of clusters obtain by deep embedded clustering. There are some clusters with very small size. These clusters can contain rare time series shapes. On the other hand, the large clusters contain the most frequent patterns.

To illustrate similarity of data points in each cluster, two data points are selected from cluster 20 and 30. In Fig. 8, it shows the similarity of selected data points in same clusters. Each cluster contains the most similar time series data.

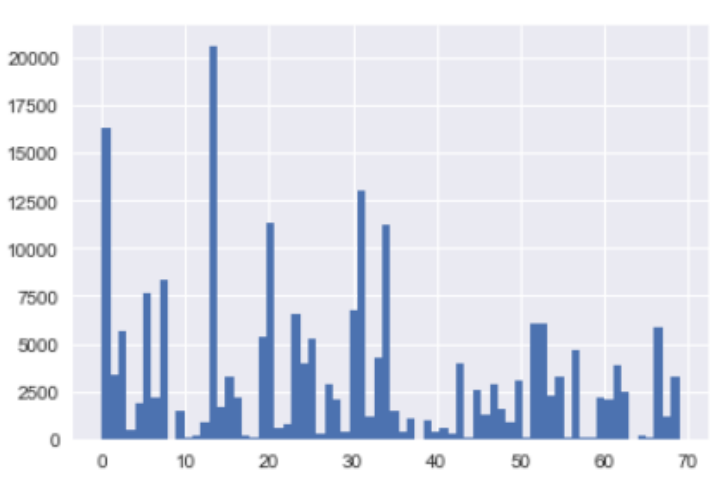

Figure 7: The histogram of size of temporal clusters.

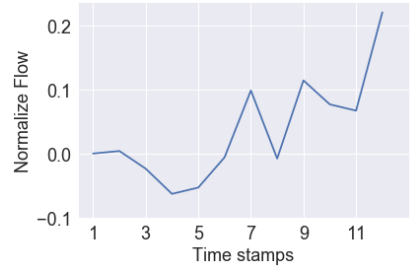

(a)

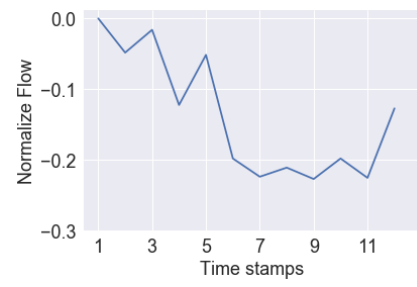

(c)

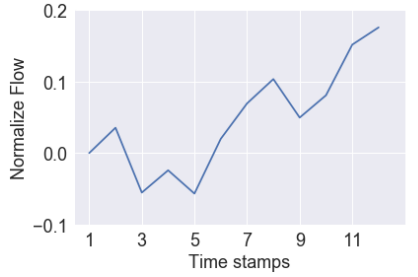

(b)

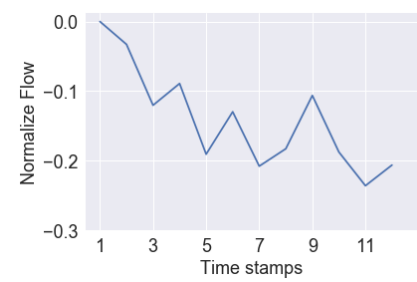

(d)
Figure 8: Four sampled data points are selected. The Fig.a and Fig.b show the data points in cluster 20. Fig.c and Fig.d show the data points in cluster 30 .

\subsection{Spatial Clusters}

In the previous section, we thoroughly analyze the temporal clusters. The analysis shows that there is clearly an interesting relation between latent feature clustering and dynamic time warping distance. Also, it shows that there is meaningful temporal patterns in the clusters. These analyses are the primary work for finding spatial clusters, because in Algorithm 1, we use temporal clusters for finding spatial clusters. In this section, we analyze the spatial clusters obtained by Algorithm 1.

We consider 70 clusters in deep embedded clustering. Each sensor $i$ has $\bar{t}$ data points, obtained by sliding window approach. The number of clusters to which each sensor is assigned has a mean of 40.4 and standard deviation of 6.5. In other words, the data points of each sensor are only assigned to $57 \%$ of the clusters on average. The analysis shows that each sensor is only assigned to part of 

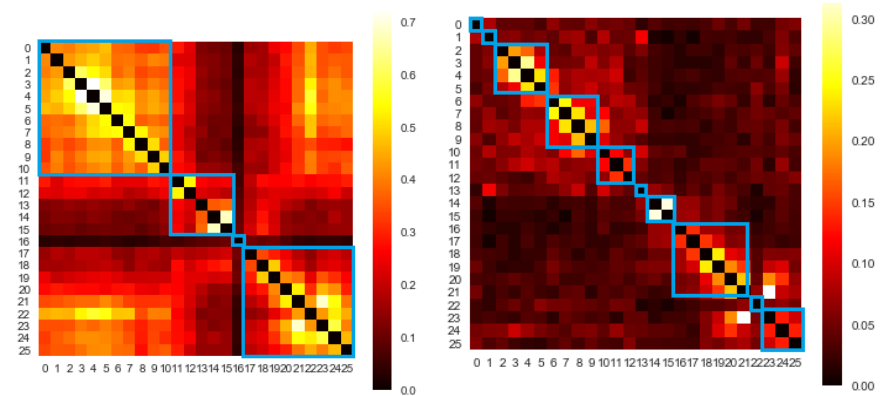

(a) Similarity of sensors in one highway(b) Similarity of sensors in one highway in in off-peak hours, 3AM. peak hours, $5 \mathrm{pm}$.

Figure 9: Heatmap of similarity matrix of sensors and the spatial clusters represented with blue rectangles.

the clusters, and it shows the output of clustering is different over spatial areas.

Algorithm 1 finds spatial clusters by comparing temporal clusters. The output of algorithm is a similarity matrix $\mathbf{C}^{\mathbf{T}}$ for a time interval $\mathbf{T}$. In the first analysis we divided traffic data into two time intervals. Figure 9 shows the similarity matrix with a heat-map. The result is presented for off-peak hours and peak hours. The output shows that in off-peak hours sensors are highly similar in all areas. However, in peak hours, only neighboring sensors are similar to each other. In other words, most part of the highway have similar flow patterns in off-peak hours. However, traffic congestion propagates flow in neighboring areas in peak hours. It increases dissimilarity between far sensors. This property shows that the number of clusters reduces in off-peak hours and increases in peak hours. To evaluate this property, we find the average value of similarity matrix in different hours of day in Fig 10. The results show that in peak hours there is more dissimilarity among far distance sensors and as a result the size of clusters are reduced.

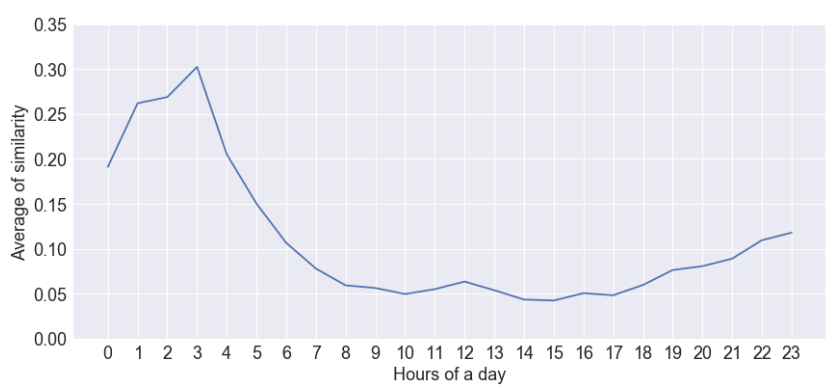

Figure 10: The relation between average similarity matrix and the hours of a day.

A threshold on similarity matrix finds the spatial cluster of neighboring similar sensors. The blue rectangles in Fig 9 represents the spatial clusters. In a similarity matrix, if higher similarities occurs near to diagonal, it shows that neighboring sensors are more similar to each other. The results show that in off-peak hours, there is more similarity in larger areas. In total, there are 4 clusters. However, in peak hours, there are 10 clusters. The neighboring sensors have more similar temporal clusters. This can be the result of flow propagation in the network, which results in higher similarity in neighboring sensors. The number of spatial clusters obtained by the proposed method over different hours of a day is in Fig 11. Higher number of clusters in peak hours shows that there is more similarities in a geographical area, when there is more flow congestion. Another interesting result is that the spatial clusters are not distributed. It shows that there is a meaningful relation between spatial similarity and distance.

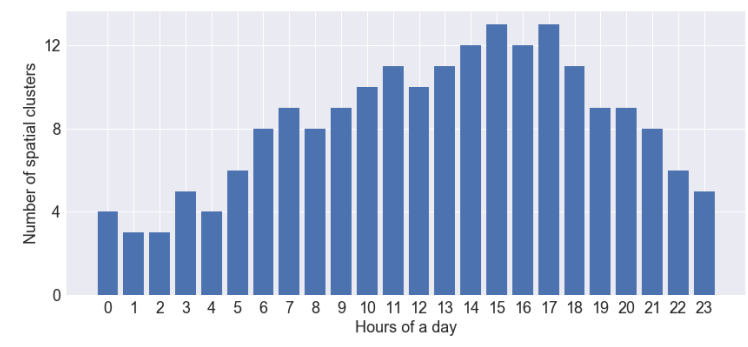

Figure 11: The number of spatial clusters for each hours of day is shown.

Further investigation can extract more insights related to spatial clustering of traffic flow. For most of the hours of the day the pattern in the highway is the same as Fig 12. In large number of time intervals in peak hours, the sensors number 16 and 22 are distinguishable from the neighboring sensors. They have their own cluster. While we only consider mainline sensors in dataset, we found both of the sensors have two off-ramps and on-ramps before and after. It shows that their traffic patterns are affected by traffic flow comes from the outside of the highway, and they are not similar to neighboring mainline sensors. Such an analysis can extract interesting relation among highways and incoming/outgoing flows. We leave this for a related further study and a longer paper.

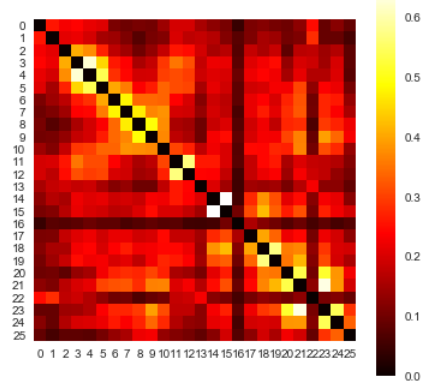

Figure 12: Heatmap of similarity of sensors. Existence of locality in traffic flow data. Similarity matrix of sensors.

\section{CONCLUSION AND FUTURE WORK}

While clustering of spatio-temporal data is a challenging and important problem, there is a lack of analysis on applying deep embedded clustering models on spatio-temporal data to investigate their corresponding patterns. In this paper, we formulate spatio-temporal 
clustering, define spatial and temporal clusters, and describe an approach in finding such clusters. To find a more efficient way of clustering, we use a deep embedded clustering model, which clusters latent feature representation of time series data. A deep analysis of temporal and spatial clusters show that they preserve temporal and spatial patterns. We describe several interesting patterns extracted from the clusters, such as high correlation between euclidean distance of latent features and DTW distance of time series, distinguishable clustering probabilities for different time stamps, and dynamic spatial clustering for various hours of a day. Unlike most of the application of time series clustering, here a dynamic spatial clustering can be more effective, when use such clusters for other analysis or machine learning models.

The proposed approach finds temporal and spatial clusters for spatio-temporal problems. There are some advantages of applying deep embedded clustering method compared with k-means clustering method. First, we describe that the computational time of deep embedded clustering is lower than k-means clustering. Also, while applying k-means clustering method on temporal data requires to define a non-linear distance function, a deep embedded clustering method do not require to have a non-linear distance function, since latent feature representations have a direct correlation with DTW method. Moreover, the deep embedded clustering method has a probabilistic output, which can be useful when the objective is to find fuzzy clusters.

This clustering approach can improve the performance of machine learning models in various problems, such as missing data imputation, forecasting and anomaly detection problems. For example, spatial clustering can find the most similar sensors in a region, and can be used for imputing missing data, or the distance of data points from temporal cluster centers can be used to detect anomalies. Moreover, the clustering of traffic data can help researchers to have further pattern analysis. Other types of neural networks, such as convolutional-recurrent neural, networks for finding an appropriate latent feature representation and other clustering models, such as agglomerative clustering model, can be investigated to better represent the clustering of spatio-temporal data. Also, this approach can find spatial and temporal clusters for other spatiotemporal data, such as weather data and power grid network data.

\section{ACKNOWLEDGEMENTS}

Reza Asadi was partially supported during this period of work by the Pacific Southwest Region University Transportation Center. We are very grateful for that support. Amelia Regan was on sabbatical during the period of this study and she is very grateful to the UCI Department of Computer Science and the University of California System for the time to devote herself to research.

\section{REFERENCES}

[1] G. Atluri, A. Karpatne, and V. Kumar, "Spatio-temporal data mining: A survey of problems and methods," ACM Computing Surveys (CSUR), vol. 51, no. 4, p. 83, 2018.

[2] A. M. Nagy and V. Simon, "Survey on traffic prediction in smart cities," Pervasive and Mobile Computing, vol. 50, pp. 148-163, 2018.

[3] F. Rempe, G. Huber, and K. Bogenberger, "Spatio-temporal congestion patterns in urban traffic networks," Transportation Research Procedia, vol. 15, pp. 513-524, 2016.
[4] L. Zhu, F. R. Yu, Y. Wang, B. Ning, and T. Tang, "Big data analytics in intelligent transportation systems: A survey," IEEE Transactions on Intelligent Transportation Systems, vol. 20, no. 1, pp. 383-398, 2018.

[5] C. Wang, X. Li, X. Zhou, A. Wang, and N. Nedjah, "Soft computing in big data intelligent transportation systems," Applied Soft Computing, vol. 38, pp. 1099-1108, 2016.

[6] M. Ahmed and A. N. Mahmood, "Novel approach for network traffic pattern analysis using clustering-based collective anomaly detection," Annals of Data Science, vol. 2, no. 1, pp. 111-130, 2015.

[7] W. C. Ku, G. R. Jagadeesh, A. Prakash, and T. Srikanthan, "A clustering-based approach for data-driven imputation of missing traffic data," in 2016 IEEE Forum on Integrated and Sustainable Transportation Systems (FISTS), pp. 1-6, IEEE, 2016.

[8] R. Asadi and A. Regan, "A spatial-temporal decomposition based deep neural network for time series forecasting," arXiv preprint arXiv:1902.00636, 2019.

[9] M. Y. Choong, L. Angeline, R. K. Y. Chin, K. B. Yeo, and K. T. K. Teo, "Vehicle trajectory clustering for traffic intersection surveillance," in 2016 IEEE International Conference on Consumer Electronics-Asia (ICCE-Asia), pp. 1-4, IEEE, 2016.

[10] H. B. Celikoglu and M. A. Silgu, "Extension of traffic flow pattern dynamic classification by a macroscopic model using multivariate clustering," Transportation Science, vol. 50, no. 3, pp. 966-981, 2016.

[11] S. Kisilevich, F. Mansmann, M. Nanni, and S. Rinzivillo, "Spatio-temporal clustering," in Data mining and knowledge discovery handbook, pp. 855-874, Springer, 2009.

[12] H. Chunchun, L. Nianxue, Y. Xiaohong, and S. Wenzhong, "Traffic flow data mining and evaluation based on fuzzy clustering techniques.," International fournal of Fuzzy Systems, vol. 13, no. 4, 2011.

[13] M. A. Silgu and H. B. Celikoglu, "Clustering traffic flow patterns by fuzzy c-means method: some preliminary findings," in International Conference on Computer Aided Systems Theory, pp. 756-764, Springer, 2015.

[14] X. Huang, Y. Ye, L. Xiong, R. Y. Lau, N. Jiang, and S. Wang, "Time series k-means: A new k-means type smooth subspace clustering for time series data, Information Sciences, vol. 367, pp. 1-13, 2016.

[15] J. Tang, G. Zhang, Y. Wang, H. Wang, and F. Liu, "A hybrid approach to integrate fuzzy c-means based imputation method with genetic algorithm for missing traffic volume data estimation," Transportation Research Part C: Emerging Technologies, vol. 51, pp. 29-40, 2015

[16] S. Aghabozorgi, A. S. Shirkhorshidi, and T. Y. Wah, "Time-series clustering-a decade review," Information Systems, vol. 53, pp. 16-38, 2015.

[17] S. Soheily-Khah, A. Douzal-Chouakria, and E. Gaussier, "Generalized k-meansbased clustering for temporal data under weighted and kernel time warp," Pattern Recognition Letters, vol. 75, pp. 63-69, 2016.

[18] J. Paparrizos and L. Gravano, "Fast and accurate time-series clustering," ACM Transactions on Database Systems (TODS), vol. 42, no. 2, p. 8, 2017.

[19] S. Wang, J. Cao, and P. S. Yu, "Deep learning for spatio-temporal data mining: A survey," arXiv preprint arXiv:1906.04928, 2019.

[20] R. Asadi and A. Regan, "A convolution recurrent autoencoder for spatio-temporal missing data imputation," arXiv preprint arXiv:1904.12413, 2019.

[21] E. Min, X. Guo, Q. Liu, G. Zhang, J. Cui, and J. Long, "A survey of clustering with deep learning: From the perspective of network architecture," IEEE Access, vol. 6, pp. 39501-39514, 2018.

[22] J. Xie, R. Girshick, and A. Farhadi, "Unsupervised deep embedding for clustering analysis," in International conference on machine learning, pp. 478-487, 2016.

[23] X. Guo, L. Gao, X. Liu, and J. Yin, "Improved deep embedded clustering with local structure preservation.," in IfCAI, pp. 1753-1759, 2017.

[24] B. Yang, X. Fu, N. D. Sidiropoulos, and M. Hong, "Towards k-means-friendly spaces: Simultaneous deep learning and clustering," in Proceedings of the 34th International Conference on Machine Learning-Volume 70, pp. 3861-3870, JMLR. org, 2017.

[25] P. Tzirakis, M. A. Nicolaou, B. Schuller, and S. Zafeiriou, "Time-series clustering with jointly learning deep representations, clusters and temporal boundaries,"

[26] P. Vincent, H. Larochelle, I. Lajoie, Y. Bengio, and P.-A. Manzagol, "Stacked denoising autoencoders: Learning useful representations in a deep network with a local denoising criterion," fournal of machine learning research, vol. 11, no. Dec, pp. 3371-3408, 2010.

[27] "California. pems, http://pems.dot.ca.gov/, 2017,"

[28] L. v. d. Maaten and G. Hinton, "Visualizing data using t-sne," fournal of machine learning research, vol. 9, no. Nov, pp. 2579-2605, 2008. 